Psychiatry in the Health Services and Population Research Department, Institute of Psychiatry, London.

\section{References}

1 Mitchell AJ, Selmes T. Why don't patients take their medicine? Reasons and solutions in psychiatry. Adv Psychiatr Treat 2007; 13: 336-46.

2 Cooper C, Bebbington $P$, King M, Brugha T, Meltzer $H$, Bhugra $D$, et al. Why people do not take their psychotropic drugs as prescribed: results of the 2000 National Psychiatric Morbidity Survey. Acta Psychiatr Scand 2007; 116: 47-53.

3 Pratt SI, Mueser KT, Driscoll M, Wolfe R, Bartels SJ. Medication nonadherence in older people with serious mental illness: prevalence and correlates. Psychiatr Rehabil J 2006; 29: 299-310.

4 Knapp M, King D, Pugner K, Lapuerta P. Non-adherence to antipsychotic medication regimens: associations with resource use and costs. $\mathrm{Br}$ Psychiatry 2004; 184: 509-16.

5 Nosé M, Barbui C, Gray R, Tansella M. Clinical interventions for treatment non-adherence in psychosis: meta-analysis. $\mathrm{Br} J$ Psychiatry 2003; 183: 197-206

6 Department of Health. National Service Framework for Older People. Department of Health, 2001.
7 Department of Health. Prescriptions Dispensed in the Community: Statistics for 1989-99 - England. Statistical Bulletin, 2000.

8 National Prescribing Centre. Prescribing for the older person. MeReC Bulletin 2000; 11: 37-40

9 Marinker M. From Compliance to Concordance: Achieving Shared Goals in Medicine Taking. Royal Pharmaceutical Society of Great Britain, 1997.

10 Wetherell $\mathrm{JL}$, Unutzer J. Adherence to treatment for geriatric depression and anxiety. CNS Spectr 2003; 8 (12 suppl 3): 48-59.

11 Lowe CJ, Raynor DK, Courtney EA, Purvis J, Teale C. Effects of self medication programme on knowledge of drugs and compliance with treatment in elderly patients. BMJ 1995; 310: 1229-31.

12 Brown KW, Billcliff N, McCabe E. Informed consent to medication in long-term psychiatric in-patients. Psychiatr Bull 2001; 25: 132-4.

13 Folstein M, Folstein S, McHugh P. 'Mini-Mental State', a practical method for grading the cognitive state of patients for the clinician. J Psychiatr Res 1975; 12: 189-98.

14 World Health Organization. The ICD-10 Classification of Mental and Behavioural Disorders: Clinical Descriptions and Diagnostic Guidelines. WHO, 1992.

15 Chow S, Ruskey F. Drawing area-proportional Venn and Euler diagrams. In Graph Drawing (ed G Liotta): 466-77. Springer, 2004.

\title{
Stability of the diagnosis of first-episode drug-induced psychosis
}

\author{
Aravind Komuravelli, ${ }^{1}$ Rob Poole, ${ }^{2,3}$ Robert Higgo ${ }^{4}$
}

The Psychiatrist (2011), 35, 224-227, doi: 10.1192/pb.bp.110.032524

${ }^{1}$ Whiston Hospital, Prescott; ${ }^{2}$ Glyndwr University, Wrexham; ${ }^{3}$ Wrexham Academic Unit; ${ }^{4}$ Liverpool Assertive Outreach Team

Correspondence to Aravind Komuravelli

(aravind.komuravelli@5bp.nhs.uk)

First received 6 Sep 2010, final revision 24 Nov 2010, accepted 10 Dec 2010
Aims and method To assess the stability of the diagnosis of first-episode druginduced psychosis over a follow-up period of at least 2 years. Patients with no psychiatric history who had been discharged from in-patient care between January 2002 and April 2006 with a firm diagnosis of drug-induced psychosis were identified. Follow-up information for at least the next 2 years or until discharge from mental health services was collected retrospectively from psychiatric records.

Results Nearly all of the patients who remained under psychiatric follow-up had a change in diagnosis, most commonly to a schizophreniform disorder. Those who were retained in follow-up had significantly longer index admissions than those discharged to primary care $(P=0.05)$

Clinical implications This study suggests that many individuals diagnosed with drug-induced psychosis are further diagnosed as having a functional psychosis, usually schizophreniform in nature. This is compatible with the suggestion that individuals diagnosed with drug-induced psychosis are experiencing either the effects of drug intoxication or an ordinary functional psychosis complicated by incidental drug use.

Declaration of interest None.
There is continuing interest among researchers and policymakers in the relationship between cannabis use and schizophrenia. $^{1-3}$ This has revived interest in the nature of the conditions that attract the clinical diagnosis of druginduced psychosis. ${ }^{4}$ The concept of drug-induced psychosis has been criticised ${ }^{5}$ and it remains unclear whether the diagnosis reflects a valid diagnostic category or whether it is based on arbitrary assumptions regarding the causation of symptoms. A systematic review concluded that the inference of causation is not secure and that the condition 
would be better understood in terms of an association. ${ }^{6}$ One possible criterion for the validity of a diagnostic category is stability during periods of follow-up, at least in the short term. This study aims to assess the extent to which the clinical diagnosis of drug-induced psychosis remains stable over a follow-up period of at least 2 years.

\section{Method}

The study was conducted in a large National Health Service mental health trust in the north-west of England, with a predominantly urban catchment area. It contains areas of urban social deprivation as well as more prosperous suburbs and coastal towns.

The trust's IT department records ICD-10 diagnoses ${ }^{7}$ on all patients under the care of the mental health service. After an in-patient admission, diagnosis is recorded by the patient's psychiatrist, normally the consultant. Where there is no ICD-10 code recorded in the discharge summary, trained coders assign a code on the basis of the clinical diagnosis. In cases of ambiguity, coders clarify the diagnosis by contacting the treating clinician.

The IT system was used to identify all patients experiencing first-episode drug-induced psychosis (ICD-10 codes F10.5-F19.5) following an in-patient admission ending between January 2002 and April 2006. Clinical information about each patient was then gathered by two researchers (A.K. and R.H.) from the IT computer system and from mental health service case records, with particular reference to alterations in diagnosis. Data were collected from May 2008, generating follow-up data for a minimum of 2 years or until discharge from specialist mental health services.

Patients were excluded from the study if they had been given any psychiatric diagnosis before the index diagnosis of drug-induced psychosis, if they had been unintentionally lost to follow-up within 2 years of the index admission (e.g. if they moved away from the area) or if there was insufficient information in their case records to assess the stability of the diagnosis (e.g. where clinical records had been lost). The most recent clinical diagnosis made in the case record by a senior psychiatrist was regarded as the 'outcome diagnosis'.

\begin{tabular}{|c|c|c|}
\hline ICD-10 coding & Psychotic disorder due to the use of & $n(\%)$ \\
\hline F12.5 & Cannabis & $32(41)$ \\
\hline F19.5 & Multiple drugs & $22(28)$ \\
\hline F14.5 & Cocaine & $14(18)$ \\
\hline F15.5 & Amphetamines and other stimulants & $6(8)$ \\
\hline F10.5 & Alcohol & $3(4)$ \\
\hline F16.5 & Hallucinogens & $1(1)$ \\
\hline
\end{tabular}

\section{Results}

Overall, 98 in-patients were diagnosed with drug-induced psychosis in the study period. Twenty were excluded from analysis: ten had a previous psychiatric diagnosis, four were lost to follow-up because they were sent to prison and one was lost because they moved away, and there was insufficient clinical information available on five patients. The initial ICD-10 diagnoses of patients included in the study are set out in Table 1.

Of the 78 patients included in the analysis, 55 were male and 23 were female. Thirty-two patients (41\%) were discharged to general practitioner (GP) care within 2 years of diagnosis and only one of these patients was re-referred to specialist mental health services after discharge to GP.

At the time of data collection 46 patients were still in follow-up ('retained patients'). This generated follow-up periods of between 2 and 6 years. Only five patients had an outcome diagnosis of drug-induced psychosis. In the case of a further five patients the outcome diagnosis was unclear from the clinical notes. Just over half of the retained patients ( $31 \%$ of patients included in the study) had a firm outcome diagnosis of schizophrenia or schizoaffective disorder. Outcomes at each stage in the study are summarised in Fig. 1 and the outcome diagnoses of the retained group are shown in Table 2 .

Mean duration of the index admission for patients subsequently discharged to primary care follow-up was 14.4 days (median 11, range 1-54) and for patients retained in follow-up it was 20.4 days (median 16, range 1-76). This difference is statistically significant (Mann-Whitney $U$-test, $P=0.05)$.

Thirty-two of the patients were diagnosed with psychosis induced by cannabis and 23 of these were retained in follow-up. Only one had an outcome diagnosis of drug-induced psychosis and in four cases there was no clear diagnosis. The most common outcome diagnosis was schizophrenia.

Further, 46 patients were diagnosed with psychosis induced by drugs other than cannabis or by poly-drug misuse; 23 were retained in follow-up. Four had a current diagnosis of drug-induced psychosis and 18 had a different current diagnosis, predominantly schizophreniform disorder. In one case there was no clear current diagnosis.

\begin{tabular}{llc}
\hline Table 2 & $\begin{array}{l}\text { Outcome diagnosis of patients retained } \\
\text { in follow-up }(n=46)\end{array}$ & $\begin{array}{c}\text { Percentage of total } \\
\text { sample }(N=78), \%\end{array}$ \\
Diagnosis & 18 & 23 \\
\hline Schizophrenia & 6 & 8 \\
\hline Schizoaffective disorder & 5 & 6 \\
\hline Drug-induced psychosis & 4 & 5 \\
\hline Bipolar affective disorder & 4 & 5 \\
\hline Psychosis not otherwise specified & 3 & 4 \\
\hline Acute and transient psychosis & 1 & 1 \\
\hline Delusional disorder & 5 & 6 \\
\hline Diagnosis unclear & 4 & \\
\hline
\end{tabular}




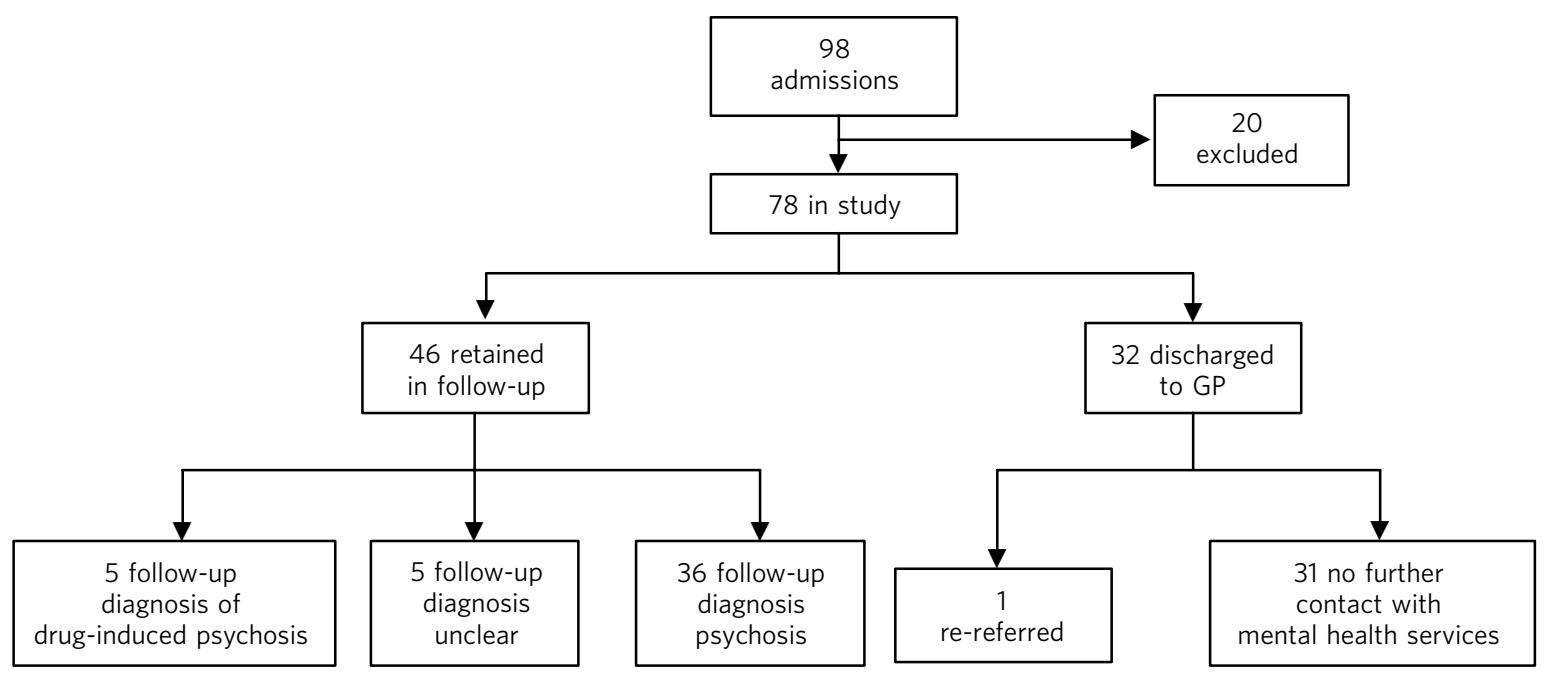

Fig 1 Flow chart of patient outcomes. GP, general practitioner.

Of the 22 patients who were diagnosed with psychosis due to poly-drug misuse, 12 were retained in follow-up. Three had a current diagnosis of drug-induced psychosis whereas nine had a current diagnosis of a different psychosis, predominantly schizophreniform disorder.

There was no statistically significant difference in the proportion of patients retained in follow-up between psychosis attributed to cannabis, other drugs or poly-drug misuse (non-parametric $\chi^{2}, P=0.129$ ).

The use of the diagnosis of drug-induced psychosis increased during the study period. The rate of new diagnosis from 2002 to 2004 appeared stable at approximately 1 per month (s.d. $=0.6)$. In 2005 this increased to 2.5 per month. The rate for the first 4 months of 2006 was 3 per month. It should be noted that a new service for early intervention with adolescents at risk of psychosis became operational in March 2005. The proportion of patients that were retained in follow-up for at least 2 years increased in the later period: from 2002 to $2004,48.6 \%$ were retained in follow-up, but among patients diagnosed after 1 January 2005, 67.4\% were retained in follow-up.

\section{Discussion}

\section{Comparison with other studies}

Our findings are consistent with two similar studies, one from the $\mathrm{USA}^{8}$ and one from the UK. ${ }^{9}$ Our follow-up was longer than that in the US study and our sample was larger than the sample in the UK study, although both studies had methodological advantages over ours. Our findings suggest that in-patients diagnosed with drug-induced psychosis on first presentation to mental health services fall into two groups of approximately equal size. The first group appear to make a prompt recovery and, once discharged by services, are not re-referred for specialist care over the next few years. The second group have a longer index admission, are retained in long-term follow-up, and are very likely to have a change in diagnosis to a functional psychosis, most commonly schizophrenia.
Our findings are also consistent with those reported in a large study of cannabis-induced psychosis using data from the Danish Psychiatric Central Register. ${ }^{10}$ Compared with that study, our sample is relatively small, but it does allow some comparison of episodes attributed solely to cannabis with other drug-induced psychoses. Although there was a non-significant trend for more patients with cannabisinduced psychosis to remain in follow-up, half of the patients where a different drug, or poly-drug misuse, was implicated also remained in follow-up. There was no statistically significant difference between the patients with cannabis-induced psychosis and other drug-induced psychosis in the pattern of diagnostic change when those patients were retained in follow-up.

It is well established that there is an association between cannabis use and schizophrenia. ${ }^{11,12}$ However, the causal link between drug use and drug-induced psychosis rests on an assumption of causality made by the clinician and that assumption may be wrong. It is possible that the initial presentation in the retained group was causally related to drug use, and that the disorder then evolved into persistent functional psychosis. It is equally possible that the patients who were eventually diagnosed with functional psychosis were actually experiencing that disorder all along, and that drug use was simply a complicating factor. In contrast with many other authors who have written on this subject, we believe that the latter interpretation is as plausible as the former, and should be taken just as seriously as a hypothesis. It has the advantage of compatibility with Occam's razor.

\section{Limitations}

There remains a large group, roughly half of our sample, who were discharged from follow-up. The major limitation of our study is that we collected very limited information on these individuals after discharge to primary care - all we could record was their utilisation of local secondary mental health services. This group tended to have shorter admissions. It is impossible to make a definitive statement 
regarding clinical outcome for them. However, it seems unlikely that many of them had a further acute and florid psychotic episode during the study period. The most plausible explanation for their index episode is that they experienced transient psychotic symptoms due to intoxication with drugs and that the symptoms subsided once the drug was eliminated. If this inference is correct, then these individuals did not experience drug-induced psychosis but acute drug intoxication.

Our study has some further limitations. Data were collected post hoc from case notes and the clinical IT system. We did not collect comparative data regarding the stability of diagnosis in patients who had not taken drugs. Of the original 98 patients, 10 were excluded because there was inadequate information available. In a further five cases, the outcome diagnosis was unclear. However, it seems unlikely that these limitations or causes of missing data created a bias that would invalidate our principle findings.

It was suggested more than a decade ago that the clinical diagnosis of drug-induced psychosis is heterogeneous, based on an inference regarding causality of uncertain validity and potentially misleading. ${ }^{5}$ Our interpretation of our findings, together with similar studies, would tend to support these assertions. It was also predicted that, despite these problems, the diagnosis was likely to continue to be used. Our study clearly confirms this prediction and even shows that the use of the diagnosis may be increasing.

\section{Clinical implications}

The main clinical implication of our study is that a significant proportion of patients presenting with apparent drug-induced psychosis experience long-term mental health problems, and that this may be especially true when initial psychotic symptoms are slow to resolve. This suggests that psychosis should be attributed to drug misuse with caution and that the expectation that further episodes can necessarily be prevented solely through avoidance of drug misuse is unrealistic in a high proportion of cases.

\section{Acknowledgement}

We are grateful to Sarah Jennings for her assistance with the statistical analysis.

\section{About the authors}

Aravind Komuravelli is specialty trainee registrar year 6, Knowsley Resource and Recovery Centre, Whiston Hospital, Prescott; Rob Poole is Professor of Mental Health, Glyndŵr University, Wrexham, and honorary consultant psychiatrist, Betsi Cadwaladr University Health Board, Wrexham Academic Unit, Wrexham; Robert Higgo is consultant psychiatrist, Liverpool Assertive Outreach Team, Arundel House, Liverpool.

\section{References}

1 van Os J, Bak M, Hanssen M, Bijl RV, de Graaf R, Verdoux H. Cannabis use and psychosis: a longditudinal population based study. Am J Epidemiol 2002; 156: 319-27.

2 Zammit S, Spurlock G, Williams H, Norton N, Williams N, O'Donovan $M C$, et al. Genotype effects of CHRNA7, CNR1 and COMT in schizophrenia: interactions with tobacco and cannabis use. $\mathrm{Br} J$ Psychiatry 2007; 191: 402-7.

3 Moore T, Zammit S, Lingford-Hughes A, Barnes T, Jones P, Burke M, et al. Cannabis use and risk of psychotic or affective mental health outcomes: a systematic review. Lancet 2007; 370: 319-28.

4 Curran C, Byrappa N, McBride A. Stimulant psychosis: a systematic review. Br J Psychiatry 2004; 185: 196-204.

5 Poole R, Brabbins C. Drug induced psychosis. Br J Psychiatry 1996; 168 135-8.

6 Mathias S, Lubman D, Hides L. Substance-induced psychosis: a diagnostic conundrum. J Clin Psychiatry 2008; 69: 358-67.

7 World Health Organization. The ICD-10 Classification of Mental and Behavioural Disorders. WHO, 1992.

8 Caton CLM, Hasin DS, Shrout PE, Drake RE, Dominguez B, First MB, et al. Stability of early-phase primary psychotic disorders with concurrent substance use and substance-induced psychosis. Br J Psychiatry 2007; 190: 105-11.

9 Crebbin K, Mitford E, Paxton R, Turkington D. First-episode druginduced psychosis: a medium term follow up study reveals a high risk group. Soc Psychiatry Psychiatr Epidemiol 2009; 44: 710-5.

10 Arendt M, Rosenberg R, Foldager L, Perto G, Munk-Jørgensen P. Cannabis-induced psychosis and subsequent schizophrenia-spectrum disorders: follow-up study of 535 incident cases. Br J Psychiatry 2005; 187: $510-5$.

11 Regier DA, Farmer ME, Rae DS, Locke BZ, Keith SJ, Judd LL, et al. Comorbidity of mental disorders with alcohol and other drug abuse. Results from the Epidemiologic Catchment Area study. JAMA 1990; 264: 2511-8.

12 Zammit S, Allebeck P, Andreasson S, Lundberg I, Lewis G. Self-reported cannabis use as a risk factor for schizophrenia in Swedish conscripts of 1969: historical cohort study. BMJ 2002; 325: 1199-201. 